\title{
Espécies de Anastrepha Schiner (Diptera: Tephritidae), seus hospedeiros e parasitóides nos Estados do Acre e Rondônia, Brasil
}

\author{
Júlia Daniela Braga Pereira', Dayse Paes Buriti², Walkymário de Paulo Lemos \\ Wilson Rodrigues da Silva ${ }^{4}$ \& Ricardo Adaime da Silva ${ }^{5,6}$ \\ ${ }^{1}$ Universidade Federal do Amapá - UNIFAP, \\ Rod. JK, Km 02, CEP 68902-280, Macapá, AP, Brasil \\ ${ }^{2}$ Universidade Federal do Acre - UFAC, \\ Rod. BR-364, Km 04, 6637, CEP 69915-900, Rio Branco, AC, Brasil \\ ${ }^{3}$ Embrapa Amazônia Oriental - EMBRAPA, \\ Trav. Dr. Enéas Pinheiro, s/n, CEP 66095-100, Belém, PA, Brasil \\ ${ }^{4}$ Secretaria de Estado da Educação - SEED, \\ Av. FAB, s/n, Centro, CEP 68906-970, Macapá, AP, Brasil \\ ${ }^{5}$ Embrapa Amapá - EMBRAPA, \\ Rod. JK, Km 04, CEP 68902-280, Macapá, AP, Brasil \\ ${ }^{6}$ Autor para correspondência: Ricardo Adaime da Silva,e-mail: adaime@cpafap.embrapa.br
}

PEREIRA, J.D.B., BURITI, D.P., LEMOS, W.P., SILVA, W.R. \& SILVA, R.A. Species of Anastrepha Schiner (Diptera: Tephritidae), its hosts and parasitoids in the States of Acre and Rondônia, Brazil. Biota Neotrop. 10(3): http://www.biotaneotropica.org.br/v10n3/en/abstract?short-communication+bn00410032010.

\begin{abstract}
In March of 2009, samplings of fleshy fruits were accomplished in the States of Acre (counties of Brasiléia, Bujari, Capixaba, Epitaciolândia, Rio Branco, Sena Madureira, Senador Guiomard and Xapuri) and in Rondônia (Ariquemes, Candeias do Jamari, Itapuã do Oeste, Ouro Preto do Oeste and Porto Velho), aiming to improve the knowledge on diversity of fruit flies (Diptera: Tephritidae), their hosts and parasitoids. The present work constitutes the first record of Anastrepha coronilli Carrejo \& González (Tephritidae) for the State of Acre and A. coronilli, A. distincta Greene (Tephritidae), and the hymenopteran D. areolatus (Szépligeti) and O. bellus Gahan (Braconidae) for Rondônia.
\end{abstract}

Keywords: Amazon, Braconidae, Hymenoptera, fruit flies, Tephritoidea.

PEREIRA, J.D.B., BURITI, D.P., LEMOS, W.P., SILVA, W.R. \& SILVA, R.A. Espécies de Anastrepha Schiner (Diptera: Tephritidae), seus hospedeiros e parasitóides nos Estados do Acre e Rondônia, Brasil. Biota Neotrop. 10(3): http://www.biotaneotropica.org.br/v10n3/pt/abstract?short-communication+bn00410032010.

Resumo: Durante o mês de março de 2009 foram realizadas amostragens de frutos carnosos nos Estados do Acre (municípios de Brasiléia, Bujari, Capixaba, Epitaciolândia, Rio Branco, Sena Madureira, Senador Guiomard e Xapuri) e Rondônia (Ariquemes, Candeias do Jamari, Itapuã do Oeste, Ouro Preto do Oeste e Porto Velho) visando ampliar o conhecimento sobre a diversidade de moscas-das-frutas (Diptera: Tephritidae), seus hospedeiros e parasitóides. Este trabalho constitui o primeiro registro de Anastrepha coronilli Carrejo \& González (Tephritidae) para o Estado do Acre, e das espécies A. coronilli, A. distincta Greene (Tephritidae), e dos himenópteros Doryctobracon areolatus (Szépligeti) e Opius bellus Gahan (Braconidae) para Rondônia.

Palavras-chave: Amazônia, Braconidae, Hymenoptera, moscas-das-frutas, Tephritoidea. 


\section{Introdução}

O conhecimento sobre moscas-das-frutas (Diptera: Tephritidae) na região Norte do Brasil aumentou consideravelmente desde a década de 1990, quando os estudos passaram a ser realizados com mais frequência e novos registros de espécies foram feitos na região por Silva (1993), Canal Daza et al. (1994, 1995), Leonel Junior et al. (1995), Ronchi-Teles (2000), Silva \& Ronchi-Teles (2000), Carvalho (2002, 2003) e Carvalho \& Malavasi (2003). No entanto, pesquisas relacionadas ao grupo concentraram-se somente em alguns estados, particularmente Amazonas e Amapá. Para os Estados do Acre e Tocantins, até o ano 2000 não havia registros de espécies de moscas-das-frutas.

Em 2003 foram realizados os primeiros registros de tefritídeos no Acre, em levantamento utilizando armadilhas (frascos caçamoscas) em pomares de citros e fruteiras tropicais. Cinco espécies foram identificadas: Anastrepha distincta Greene, A. leptozona Hendel, A. striata Schiner, A. obliqua (Macquart) e A. tumida Stone (Thomazini et al. 2003). No referido trabalho, não foi possível a identificação dos hospedeiros das espécies obtidas, visto que essa associação somente pode ser determinada por meio da amostragem de frutos (Nascimento et al. 2000).

No Estado de Rondônia, desde o ano 2000 não há incremento no número de espécies conhecidas, sendo registradas para esse estado as espécies A. atrigona Hendel, A. leptozona, A. obliqua, A. serpentina (Wiedemann) e A. striata, de acordo com Zucchi (2008). Dessa forma, o presente trabalho objetivou registrar a ocorrência de espécies de Anastrepha, seus hospedeiros e parasitóides em municípios dos Estados do Acre e Rondônia, Brasil.

\section{Materiais e Métodos}

Foram realizadas coletas de frutos carnosos, silvestres e cultivados, em diferentes formações vegetais, de 16 a 27/03/2009 no Acre, municípios de Brasiléia, Bujari, Capixaba, Epitaciolândia, Rio Branco, Sena Madureira, Senador Guiomard e Xapuri; e de 18/03 a 21/03/2009 em Rondônia, municípios de Ariquemes, Candeias do Jamari, Itapuã do Oeste, Ouro Preto do Oeste e Porto Velho. A amostragem foi realizada ao acaso, coletando-se frutos maduros ou em estado de maturação, diretamente das plantas ou do solo, quando estes estavam recém-caídos. Todos os pontos de coleta tiveram suas coordenadas geográficas registradas por GPS. As amostras foram acondicionadas em recipientes plásticos (vedados por tecido de malha fina), devidamente identificadas, pesadas e posteriormente foram transportadas para o Laboratório de Entomologia da Embrapa Acre, onde o experimento foi conduzido, segundo Silva et al. (2007).

Os exemplares de Tephritidae e Braconidae obtidos nas coletas estão depositados no Laboratório de Entomologia da Embrapa Amapá, onde uma coleção de referência está sendo estruturada.

\section{Resultados e Discussão}

No estado do Acre foram coletadas 88 amostras, totalizando 1.976 frutos $(52,12 \mathrm{~kg}$ ) (Tabela 1$)$. Foram obtidos 723 pupários, emergindo desse total 267 tefritídeos e 5 parasitóides (Tabela 2), tendo sido os frutos de goiaba os mais infestados, com 263,6 pupários $/ \mathrm{kg}$. As espécies registradas foram A. coronilli, A. distincta, A. obliqua e A. striata (Tabela 3), sendo esta última a espécie mais frequente. Somente o parasitóide braconídeo Doryctobracon areolatus (Szépligeti) foi verificado associado a $A$. coronilli em frutos de goiaba-de-anta (Melastomataceae), totalizando 10,2\% de parasitismo (Tabela 2). Os primeiros registros de parasitóides de Anastrepha para o Estado do Acre foram feitos recentemente, sendo Opius bellus Gahan, $D$. areolatus e Utetes anastrephae (Viereck) associados a A. obliqua em frutos de taperebá, no município de Bujari, e D. areolatus a A. obliqua em frutos de goiaba, na capital Rio Branco (Thomazini \& Albuquerque 2009). Das cinco espécies de Anastrepha já registradas no Acre, capturadas com armadilhas, apenas A. leptozona e A. tumida não foram obtidas neste trabalho. Foi verificada a ocorrência de $A$. coronilli no município de Capixaba (Tabela 3), sendo este o primeiro registro da espécie para o Estado do Acre.

Em Rondônia foram coletadas 61 amostras nos cinco municípios amostrados, totalizando 1.402 frutos $(51,70 \mathrm{~kg})$ (Tabela 4). Foram obtidos 563 pupários, dos quais emergiram 78 tefritídeos e 21 parasitóides braconídeos (Tabela 5) das espécies $D$. areolatus e $O$. bellus (Tabela 6). Os frutos de araçá-goiaba foram os mais infestados $(298,2$ pupários $/ \mathrm{kg})$. D. areolatus foi o parasitóide predominante, com índice de parasitismo de 22,0\% em amostras de carambola, no município de Ouro Preto do Oeste (Tabela 5). As espécies de moscas-das-frutas registradas foram A. atrigona, A. coronilli, A. distincta, A. obliqua e A. striata (Tabela 6), sendo A. striata a mais frequente, assim como observado nas coletas realizadas no Estado do Acre. A. coronilli foi associada a frutos de goiaba (Myrtaceae) e goiaba-de-anta (Melastomataceae) e A. distincta a frutos de ingá-cipó (Fabaceae). Os resultados obtidos caracterizam o primeiro registro dos tefritídeos A. coronilli e A. distincta e dos braconídeos $D$. areolatus e $O$. bellus para o Estado de Rondônia, Brasil. A ocorrência de A. coronilli já foi registrada para os Estados do Amazonas, Amapá, Roraima e Tocantins, sendo associada a hospedeiros das famílias Annonaceae, Dileniaceae, Memecylaceae e, preferencialmente, Melastomataceae (Zucchi 2008). Este trabalho relata o primeiro registro da família Myrtaceae como hospedeiro de A. coronilli. De acordo com Zucchi (2008), A. distincta estava distribuída em todos os Estados da região Norte, exceto Rondônia. A ocorrência dos parasitóides $D$. areolatus e $O$. bellus já foi reportada na literatura para o vizinho Estado do Amazonas, nas proximidades de Manaus, sendo este o local de ocorrência mais próximo de Rondônia (Costa et al. 2009).

Tabela 1. Espécies, número e massa de frutos coletados no Estado do Acre, Brasil. Março de 2009.

Table 1. Species, number and mass of fruits collected in the State of Acre, Brazil. March 2009.

\begin{tabular}{|c|c|c|c|c|c|c|c|c|c|c|c|c|c|c|c|c|c|c|c|c|c|c|c|c|}
\hline \multirow{2}{*}{$\begin{array}{c}\text { Família } \\
\text { Nome Vernacular } \\
\text { Nome Científico }\end{array}$} & \multicolumn{3}{|c|}{ Brasiléia } & \multicolumn{3}{|c|}{ Bujari } & \multicolumn{3}{|c|}{ Capixaba } & \multicolumn{3}{|c|}{ Epitaciolândia } & \multicolumn{3}{|c|}{ Rio Branco } & \multicolumn{3}{|c|}{$\begin{array}{c}\text { Sena } \\
\text { Madureira }\end{array}$} & \multicolumn{3}{|c|}{$\begin{array}{c}\text { Senador } \\
\text { Guiomard }\end{array}$} & \multicolumn{3}{|c|}{ Xapuri } \\
\hline & $\mathrm{C} / \mathrm{I}$ & $\begin{array}{l}\mathbf{F} \\
(\mathbf{n})\end{array}$ & $\begin{array}{l}\text { M } \\
(\mathrm{g})\end{array}$ & $\mathrm{C} / \mathrm{I}$ & $\begin{array}{l}\mathbf{F} \\
(\mathbf{n})\end{array}$ & $\begin{array}{l}\text { M } \\
(\mathrm{g})\end{array}$ & $\mathrm{C} / \mathrm{I}$ & $\begin{array}{c}\mathbf{F} \\
(\mathbf{n})\end{array}$ & $\begin{array}{l}\text { M } \\
(\mathrm{g})\end{array}$ & $\mathrm{C} / \mathrm{I}$ & $\begin{array}{l}\mathbf{F} \\
(\mathbf{n})\end{array}$ & $\begin{array}{l}\text { M } \\
(\mathrm{g})\end{array}$ & $\mathrm{C} / \mathrm{I}$ & $\begin{array}{l}\mathbf{F} \\
(\mathbf{n})\end{array}$ & $\begin{array}{l}\text { M } \\
(\mathrm{g})\end{array}$ & $\mathrm{C} / \mathrm{I}$ & $\begin{array}{l}\mathbf{F} \\
(\mathbf{n})\end{array}$ & $\begin{array}{l}\text { M } \\
(\mathrm{g})\end{array}$ & $\mathrm{C} / \mathrm{I}$ & $\begin{array}{l}\mathbf{F} \\
(\mathbf{n})\end{array}$ & $\begin{array}{l}\text { M } \\
(g)\end{array}$ & $\mathrm{C} / \mathrm{I}$ & $\begin{array}{l}\text { F } \\
(\mathbf{n})\end{array}$ & $\begin{array}{l}\text { M } \\
(\mathrm{g})\end{array}$ \\
\hline nacardiaceae & & & & & & & & & & & & & & & & & & & & & & & & \\
\hline $\begin{array}{l}\text { Cajarana } \\
\text { (Spondias dulcis) }\end{array}$ & $1 / 1$ & 14 & 1.275 & - & - & - & - & - & - & - & - & - & - & - & - & - & - & - & - & - & - & $1 / 1$ & 5 & 400 \\
\hline $\begin{array}{l}\text { Caju } \\
\text { (Anacardium occidentale) }\end{array}$ & - & - & - & - & - & - & - & - & - & - & - & - & - & - & - & $1 / 0$ & 4 & 275 & - & - & - & & & \\
\hline $\begin{array}{l}\text { Arecaceae } \\
\text { Urucuri } \\
\text { (Attalea excelsa) }\end{array}$ & - & - & - & - & - & - & - & - & - & - & - & - & $1 / 0$ & 20 & 1.550 & $1 / 0$ & 5 & 400 & - & - & - & - & - & - \\
\hline
\end{tabular}


Tabela 1. Continuação...

\begin{tabular}{|c|c|c|c|c|c|c|c|c|c|c|c|c|c|c|c|c|c|c|c|c|c|c|c|c|}
\hline \multirow{2}{*}{$\begin{array}{c}\text { Família } \\
\text { Nome Vernacular } \\
\text { Nome Científico }\end{array}$} & \multicolumn{3}{|c|}{ Brasiléia } & \multicolumn{3}{|c|}{ Bujari } & \multicolumn{3}{|c|}{ Capixaba } & \multicolumn{3}{|c|}{ Epitaciolândia } & \multicolumn{3}{|c|}{ Rio Branco } & \multicolumn{3}{|c|}{$\begin{array}{c}\text { Sena } \\
\text { Madureira }\end{array}$} & \multicolumn{3}{|c|}{$\begin{array}{c}\text { Senador } \\
\text { Guiomard }\end{array}$} & \multicolumn{3}{|c|}{ Xapuri } \\
\hline & $\mathrm{C} / \mathrm{I}$ & $\begin{array}{l}\mathbf{F} \\
(\mathbf{n})\end{array}$ & $\begin{array}{l}\mathbf{M} \\
(\mathrm{g})\end{array}$ & $\mathrm{C} / \mathrm{I}$ & $\begin{array}{l}\mathbf{F} \\
(\mathbf{n})\end{array}$ & $\begin{array}{l}\mathbf{M} \\
(\mathrm{g})\end{array}$ & $\mathrm{C} / \mathrm{I}$ & $\begin{array}{l}\mathbf{F} \\
(\mathbf{n})\end{array}$ & $\begin{array}{l}\mathbf{M} \\
(\mathrm{g})\end{array}$ & $\mathrm{C} / \mathrm{I}$ & $\begin{array}{l}\mathbf{F} \\
(\mathbf{n})\end{array}$ & $\begin{array}{l}\mathbf{M} \\
(\mathrm{g})\end{array}$ & $\mathrm{C} / \mathrm{I}$ & $\begin{array}{l}\mathbf{F} \\
(\mathbf{n})\end{array}$ & $\begin{array}{l}\mathbf{M} \\
(\mathrm{g})\end{array}$ & $\mathrm{C} / \mathrm{I}$ & $\begin{array}{l}\mathbf{F} \\
(\mathbf{n})\end{array}$ & $\begin{array}{l}\mathbf{M} \\
(\mathrm{g})\end{array}$ & $\mathrm{C} / \mathrm{I}$ & $\begin{array}{l}\mathbf{F} \\
(\mathbf{n})\end{array}$ & $\begin{array}{l}\mathbf{M} \\
(\mathrm{g})\end{array}$ & $\mathrm{C} / \mathrm{I}$ & $\begin{array}{l}\mathbf{F} \\
(\mathbf{n})\end{array}$ & $\begin{array}{l}\mathbf{M} \\
(\mathrm{g})\end{array}$ \\
\hline $\begin{array}{l}\text { Annonaceae } \\
\text { Biribá } \\
(\text { Rollinia mucosa })\end{array}$ & - & - & - & $1 / 0$ & 3 & 1.350 & $1 / 0$ & 2 & 525 & - & - & - & $5 / 0$ & 18 & 2.475 & $1 / 0$ & 4 & 1.300 & - & - & - & - & - & - \\
\hline $\begin{array}{l}\text { Graviola } \\
\text { (Annona muricata) }\end{array}$ & - & - & - & - & - & - & - & - & - & - & - & - & $1 / 0$ & 1 & 550 & - & - & - & - & - & - & - & - & - \\
\hline $\begin{array}{l}\text { Caricaceae } \\
\text { Mamão } \\
\text { (Carica papaya })\end{array}$ & - & - & - & $1 / 0$ & 2 & 3.200 & $1 / 0$ & 1 & 1.525 & $1 / 0$ & 1 & 1.050 & $1 / 1$ & 1 & 900 & - & - & - & $1 / 0$ & 1 & 700 & $1 / 0$ & 1 & 1.350 \\
\hline $\begin{array}{l}\text { Combretaceae } \\
\text { Castanhola } \\
\text { (Terminalia catappa) }\end{array}$ & - & - & - & - & - & - & - & - & - & - & - & - & $1 / 0$ & 25 & 500 & - & - & - & - & - & - & - & - & - \\
\hline $\begin{array}{l}\text { Fabaceae } \\
\text { Ingá-cipó } \\
\text { (Inga edulis) }\end{array}$ & - & - & - & $1 / 1$ & 3 & 500 & $1 / 1$ & 1 & 425 & - & - & - & - & - & - & - & - & - & - & - & - & - & - & - \\
\hline $\begin{array}{l}\text { Malpighiaceae } \\
\text { Acerola } \\
\text { (Malpighia punicifolia) }\end{array}$ & $2 / 0$ & 115 & 600 & $2 / 0$ & 109 & 425 & $3 / 0$ & 192 & 965 & $2 / 0$ & 120 & 600 & $4 / 0$ & 381 & 1.650 & - & - & - & $2 / 0$ & 90 & 400 & $2 / 0$ & 45 & 200 \\
\hline $\begin{array}{l}\text { Melastomataceae } \\
\text { Goiaba-de-anta } \\
\text { (Bellucia grossulariodes) }\end{array}$ & - & - & - & - & - & - & $1 / 1$ & 50 & 600 & - & - & - & - & - & - & - & - & - & - & - & - & - & - & - \\
\hline $\begin{array}{l}\text { Moraceae } \\
\text { Amora } \\
\text { (Morus sp.) }\end{array}$ & - & - & - & - & - & - & - & - & - & - & - & - & $1 / 0$ & 80 & 75 & - & - & - & - & - & - & - & - & - \\
\hline $\begin{array}{l}\text { Figo } \\
\text { (Ficus carica) }\end{array}$ & - & - & - & - & - & - & - & - & - & - & - & - & - & - & - & - & - & - & - & - & - & $1 / 0$ & 3 & 125 \\
\hline $\begin{array}{l}\text { Myrtaceae } \\
\text { Araçá-boi } \\
\text { (Eugenia stipitata) }\end{array}$ & - & - & - & - & - & - & $1 / 0$ & 4 & 300 & - & - & - & $1 / 1$ & 3 & 825 & - & - & - & - & - & - & - & - & - \\
\hline $\begin{array}{l}\text { Goiaba } \\
\text { (Psidium guajava) }\end{array}$ & - & - & - & - & - & - & - & - & - & $1 / 1$ & 13 & 300 & $2 / 1$ & 18 & 1.005 & $3 / 3$ & 52 & 1.950 & $2 / 2$ & 40 & 1.350 & $1 / 1$ & 7 & 225 \\
\hline $\begin{array}{l}\text { Pitanga } \\
\text { (Eugenia uniflora) }\end{array}$ & - & - & - & $1 / 0$ & 7 & 50 & - & - & - & - & - & - & - & - & - & - & - & - & $1 / 0$ & 40 & 200 & - & - & - \\
\hline $\begin{array}{l}\text { Passifloraceae } \\
\text { Maracujá } \\
\text { (Passiflora sp.) }\end{array}$ & - & - & - & - & - & - & - & - & - & - & - & - & $1 / 0$ & 6 & 425 & $1 / 0$ & 6 & 250 & - & - & - & - & - & - \\
\hline $\begin{array}{l}\text { Rubiaceae } \\
\text { Café } \\
\text { (Coffea } \mathrm{sp} .)\end{array}$ & - & - & - & $1 / 0$ & 85 & 100 & $1 / 0$ & 170 & 100 & - & - & - & - & - & - & - & - & - & - & - & - & - & - & - \\
\hline $\begin{array}{l}\text { Rutaceae } \\
\text { Laranja } \\
\text { (Citrus sinensis) }\end{array}$ & $1 / 0$ & 10 & 1.175 & $1 / 0$ & 4 & 500 & $2 / 1$ & 14 & 1.225 & - & - & - & $1 / 0$ & 5 & 550 & $1 / 0$ & 3 & 450 & - & - & - & $1 / 0$ & 6 & 1.700 \\
\hline $\begin{array}{l}\text { Lima } \\
\text { (Citrus aurantifolia) }\end{array}$ & - & - & - & $1 / 0$ & 3 & 475 & - & - & - & - & - & - & - & - & - & - & - & - & - & - & - & - & - & - \\
\hline $\begin{array}{l}\text { Tangerina } \\
\text { (Citrus nobilis) }\end{array}$ & $1 / 0$ & 6 & 800 & - & - & - & - & - & - & - & - & - & - & - & - & - & - & - & - & - & - & - & - & - \\
\hline $\begin{array}{l}\text { Sapotaceae } \\
\text { Sapoti } \\
(\text { Manilkara zapota })\end{array}$ & - & - & - & - & - & - & - & - & - & - & - & - & - & - & - & - & - & - & - & - & - & $1 / 0$ & 7 & 675 \\
\hline $\begin{array}{l}\text { Solanaceae } \\
\text { Jiló } \\
\text { (Solanum gilo) }\end{array}$ & - & - & - & - & - & - & - & - & - & - & - & - & $1 / 0$ & 20 & 500 & - & - & - & - & - & - & - & - & - \\
\hline $\begin{array}{l}\text { Oxalidaceae } \\
\text { Carambola } \\
(\text { Averrhoa carambola) }\end{array}$ & $1 / 1$ & 6 & 650 & $2 / 0$ & 16 & 875 & $2 / 2$ & 12 & 1.025 & $2 / 2$ & 21 & 2.050 & $4 / 2$ & 39 & 2.975 & $2 / 0$ & 19 & 625 & $2 / 0$ & 16 & 1.300 & $2 / 1$ & 21 & 1.600 \\
\hline
\end{tabular}

C/I: Amostras coletadas/infestadas. F: número de frutos. M: peso de frutos em gramas. 
Tabela 2. Índices de infestação por moscas-das-frutas e parasitismo registrados em amostras de frutos coletadas no Estado do Acre, Brasil. Março de 2009.

Table 2. Index of infestation by fruit flies and parasitism registrated in fruit samples from State of Acre, Brazil. March 2009.

\begin{tabular}{|c|c|c|c|c|c|c|}
\hline Município & Hospedeiro & $\begin{array}{c}\text { Pupários } \\
\text { (n) }\end{array}$ & $\begin{array}{c}\text { Infestação } \\
\text { (pupários/kg) }\end{array}$ & $\begin{array}{c}\text { Anastrepha } \\
(\mathbf{n})^{* * *}\end{array}$ & $\begin{array}{c}\text { Parasitóides } \\
(\mathbf{n})^{* * *}\end{array}$ & $\begin{array}{c}\text { Parasitismo } \\
(\%)\end{array}$ \\
\hline \multirow[t]{2}{*}{ Brasiléia } & Cajarana & 51 & 40,0 & 6 & 0 & 0 \\
\hline & Carambola & 8 & 12,3 & 0 & 0 & 0 \\
\hline Bujari & Ingá-cipó & 64 & 128 & 14 & 0 & 0 \\
\hline \multirow[t]{4}{*}{ Capixaba } & Carambola & 7 & 6,8 & 0 & 0 & 0 \\
\hline & Goiaba-de-anta & 49 & 81,7 & 6 & 5 & 10,2 \\
\hline & Ingá-cipó & 33 & 77,6 & 0 & 0 & 0 \\
\hline & Laranja & 4 & 4,8 & 0 & 0 & 0 \\
\hline \multirow[t]{2}{*}{ Epitaciolândia } & Carambola & 7 & 3,4 & 0 & 0 & 0 \\
\hline & Goiaba & 59 & 196,7 & 33 & 0 & 0 \\
\hline \multirow[t]{5}{*}{ Rio Branco } & Araçá-boi & 15 & 18,0 & 0 & 0 & 0 \\
\hline & Carambola & 9 & 6,0 & 0 & 0 & 0 \\
\hline & Goiaba & 87 & 263,6 & 20 & 0 & 0 \\
\hline & Maracujá & 5 & 11,8 & 0 & 0 & 0 \\
\hline & Mamão & 6 & 6,6 & 0 & 0 & 0 \\
\hline Sena Madureira & Goiaba & 224 & 146,9 & 146 & 0 & 0 \\
\hline Senador Guiomard & Goiaba & 91 & 92,4 & 42 & 0 & 0 \\
\hline \multirow[t]{3}{*}{ Xapuri } & Cajarana & 2 & 5,0 & 0 & 0 & 0 \\
\hline & Carambola & 1 & 0,9 & 0 & 0 & 0 \\
\hline & Goiaba & 1 & 4,4 & 0 & 0 & 0 \\
\hline Totais & - & 723 & - & 267 & 5 & - \\
\hline
\end{tabular}

*machos e fêmeas

Tabela 3. Espécies de Tephritidae e parasitóides associados a diferentes plantas frutíferas coletadas no Estado do Acre, Brasil. Março de 2009.

Table 3. Species of Tephritidae and parasitoids associated to different fruits collected in the State of Acre, Brazil. March 2009.

\begin{tabular}{|c|c|c|c|}
\hline Município & Hospedeiro & Tephritidae $(\mathbf{n})^{*}$ & Parasitóide (n) ${ }^{* *}$ \\
\hline Brasiléia & Cajarana & A. obliqua (5) & - \\
\hline Bujari & Ingá-cipó & A. distincta (13) & - \\
\hline & & A. striata (1) & \\
\hline Capixaba & Goiaba-de-anta & A. coronilli (3) & D. areolatus (5) \\
\hline Epitaciolândia & Goiaba & A. striata (18) & - \\
\hline Rio Branco & Goiaba & A. striata $(8)$ & - \\
\hline Sena Madureira & Goiaba & A. striata $(60)$ & - \\
\hline & & A. distincta (1) & \\
\hline Senador Guiomard & Goiaba & A. striata (24) & - \\
\hline
\end{tabular}

"fêmeas " machos e fêmeas

Tabela 4. Espécie, número e massa de frutos coletados no Estado de Rondônia, Brasil. Março de 2009.

Table 4. Species, number and mass of fruits collected in the State of Rondônia, Brazil. March 2009.

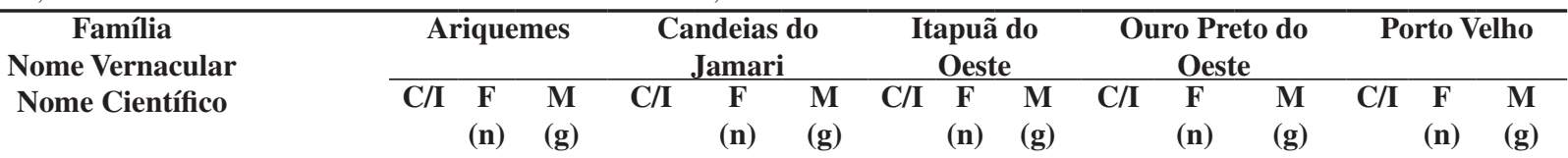

\section{Anacardiaceae}

Cajá (Spondias lutea)

\section{Annonaceae}

Araticum (Annona montana)

Biribá (Rollinia mucosa)

Graviola (Annona muricata)

Apocynaceae

Quina (Geissospermum argenteum)

Arecaceae

Pupunha (Bactris gasipaes)

Caricaceae

Mamão (Carica papaya)

Cucurbitaceae

Abóbora (Cucurbita sp.)

Euphorbiaceae

Mandioca (Manihot esculenta)

\begin{tabular}{|c|c|c|c|c|c|c|c|c|c|c|c|c|c|c|}
\hline- & - & - & - & - & - & $1 / 1$ & 6 & 500 & - & - & - & - & - & - \\
\hline- & - & - & - & - & - & - & - & - & - & - & - & $1 / 0$ & 4 & 3.700 \\
\hline $1 / 0$ & 2 & 425 & $1 / 0$ & 1 & 750 & - & - & - & $1 / 0$ & 2 & 925 & $1 / 0$ & 10 & 5.750 \\
\hline- & - & - & & & & - & - & - & - & - & - & $1 / 0$ & 1 & 1.275 \\
\hline- & - & - & - & - & - & - & - & - & $1 / 1$ & 21 & 475 & $1 / 1$ & 95 & 1.075 \\
\hline - & - & - & - & - & - & - & - & - & $1 / 0$ & 4 & 175 & - & - & - \\
\hline- & - & - & - & - & - & $2 / 0$ & 4 & 2.945 & $1 / 0$ & 1 & 1.175 & - & - & - \\
\hline - & - & - & - & - & - & $1 / 0$ & 2 & 2.450 & - & - & - & - & - & - \\
\hline & & & - & - & - & $1 / 0$ & 1 & 200 & - & - & - & - & - & - \\
\hline
\end{tabular}

C/I: Amostras coletadas/infestadas. F: número de frutos. M: peso de frutos em gramas. 
Tabela 4. Continuação..

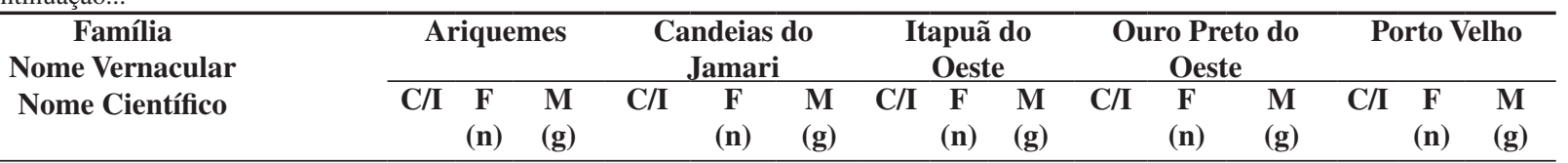

\section{Fabaceae}

Ingá-cipó (Inga edulis)

Ingazinha (Inga sp.)

Tamarindo (Tamarindus indica)

Humiriaceae

Uxi (Endopleura uchi)

Malpighiaceae

Acerola (Malpighia punicifolia)

Muruci (Byrsonima crassifolia)

Melastomataceae

Goiaba-de-anta (Bellucia grossularioides)

Myrtaceae

Araçá-boi (Eugenia stipitata)

Araçá-goiaba (Psidium guineense)

Camu-camu (Myrciaria dubia)

Goiaba (Psidium guajava)

Jambo (Syzygium jambos)

Pitanga (Eugenia uniflora)

Oxalidaceae

$\begin{array}{cccccccccccc}- & - & - & - & - & - & 2 / 2 & 15 & 5.775 & - & - & - \\ 1 / 0 & 13 & 225 & - & - & - & - & - & - & - & - & - \\ - & - & - & - & - & - & - & - & - & 1 / 0 & 15 & 275 \\ - & - & - & - & - & - & - & - & - & 1 / 0 & 4 & 275\end{array}$

Carambola (Averrhoa carambola)

Passifloraceae

Maracujá (Passiflora sp.)

Rubiaceae

Café (Coffea sp.)

Noni (Morinda citrifolia)

Rutaceae

Laranja (Citrus sinensis)

Limão (Citrus limonum)

Tangerina (Citrus nobilis)

Sapindaceae

Rambotão (Nephelium lappaceum)

$\begin{array}{lllllllllllllllll}1 / 0 & 43 & 200 & 1 / 0 & 53 & 275 & - & - & - & - & - & - & 1 / 0 & 122 & 675\end{array}$

Solanaceae

Jiló (Solanum gilo)

Jurubeba (Solanum paniculatum)

Pimenta-vermelha (Capsicum sp.)

Sterculiaceae

Cupuí (Theobroma sp.)

C/I: Amostras coletadas/infestadas. F: número de frutos. M: peso de frutos em gramas.

Tabela 5. Índices de infestação por moscas-das-frutas e parasitismo registrados em amostras de frutos coletadas no Estado de Rondônia, Brasil. Março de 2009.

Table 5. Index of infestation by fruit flies and parasitism registrated in fruit samples from State of Rondônia, Brazil. March 2009.

\begin{tabular}{|c|c|c|c|c|c|c|}
\hline Município & Hospedeiro & $\begin{array}{c}\text { Pupários } \\
\text { (n) }\end{array}$ & $\begin{array}{c}\text { Infestação } \\
\text { (pupários/kg) }\end{array}$ & $\begin{array}{c}\text { Anastrepha } \\
(\mathbf{n})^{* * *}\end{array}$ & $\begin{array}{c}\text { Parasitóides } \\
(\mathbf{n})^{* * *}\end{array}$ & $\begin{array}{c}\text { Parasitismo } \\
(\%)\end{array}$ \\
\hline \multirow[t]{2}{*}{ Ariquemes } & Carambola & 1 & 0,9 & 0 & 0 & 0 \\
\hline & Goiaba & 21 & 36,5 & 7 & 0 & 0 \\
\hline Candeias do Jamari & Carambola & 1 & 0,8 & 0 & 0 & 0 \\
\hline \multirow[t]{2}{*}{ Itapuã do Oeste } & Cajá & 6 & 12,0 & 0 & 0 & 0 \\
\hline & Ingá-cipó & 29 & 7,2 & 9 & 0 & 0 \\
\hline \multirow[t]{7}{*}{ Ouro Preto do Oeste } & Araçá-boi & 7 & 12,7 & 1 & 0 & 0 \\
\hline & Araçá-goiaba & 68 & 160,0 & 5 & 3 & 4,4 \\
\hline & Carambola & 45 & 85,7 & 5 & 10 & 22,0 \\
\hline & Goiaba & 118 & 96,3 & 31 & 5 & 4,2 \\
\hline & Goiaba-de-anta & 120 & 141,2 & 9 & 3 & 2,5 \\
\hline & Jambo & 3 & 5,5 & 0 & 0 & 0 \\
\hline & Quinarana & 3 & 6,3 & 0 & 0 & 0 \\
\hline \multirow[t]{3}{*}{ Porto Velho } & Araçá-goiaba & 82 & 298,2 & 5 & 0 & 0 \\
\hline & Goiaba-de-anta & 2 & 2,5 & 0 & 0 & 0 \\
\hline & Quina & 57 & 53,0 & 6 & 0 & 0 \\
\hline Totais & - & 563 & - & 78 & 21 & - \\
\hline
\end{tabular}

machos e fêmeas 
Pereira, J. D. B. et al.

Tabela 6. Espécies de Tephritidae e parasitóides associados em frutos coletados no Estado de Rondônia, Brasil. Março de 2009.

Table 6. Species of Tephritidae and parasitoids associated to different fruits collected in the State of Rondônia, Brazil. March 2009.

\begin{tabular}{|c|c|c|c|}
\hline Município & Hospedeiro & Tephritidae (n)* & Parasitóide (n) ${ }^{* * *}$ \\
\hline Ariquemes & Goiaba & A. striata (5) & - \\
\hline Itapuã do Oeste & Ingá-cipó & A. distincta (8) & - \\
\hline \multirow[t]{5}{*}{ Ouro Preto do Oeste } & Araçá-boi & A. obliqua (1) & - \\
\hline & Araçá-goiaba & A. striata (1) & $\begin{array}{l}\text { D. areolatus (2) } \\
\text { O. bellus (1) }\end{array}$ \\
\hline & Carambola & A. obliqua (2) & $\begin{array}{l}\text { D. areolatus (9) } \\
\text { O. bellus (1) }\end{array}$ \\
\hline & Goiaba & $\begin{array}{l}\text { A. striata }(11) \\
\text { A. atrigona }(1) \\
\text { A. coronilli }(1)\end{array}$ & D. areolatus (5) \\
\hline & Goiaba-de-anta & $\begin{array}{l}\text { A. coronilli (3) } \\
\text { A. striata (1) }\end{array}$ & D. areolatus (3) \\
\hline \multirow[t]{2}{*}{ Porto Velho } & Araçá-goiaba & A. obliqua (2) & - \\
\hline & Quina & $\begin{array}{l}\text { A. atrigona }(3) \\
\text { A. obliqua }(1)\end{array}$ & - \\
\hline
\end{tabular}

"fêmeas *"machos e fêmeas

\section{Agradecimentos}

Os autores agradecem a Carlos Alberto Moraes (Embrapa Amapá) e ao Sr. Claudir Vezu (Embrapa Acre), pelo auxílio nas coletas de frutos. Aos pesquisadores Murilo Fazolin e Givanildo Roncatto (Embrapa Acre), César Augusto Domingues Teixeira (Embrapa Rondônia) e Olzeno Trevisan (CEPLAC Rondônia), pela viabilização da logística dos trabalhos de campo.

\section{Referências Bibliográficas}

CANAL DAZA, N., ZUCCHI, R.A., SILVA, N.M. \& LEONEL Jr., F.L. 1994. Reconocimento de las especies de parasitoides (Hym.: Braconidae) de moscas de las frutas Dip.: (Tephritidae) em dos municipios del estado de Amazonas, Brasil. Bol. Mus. Entomol. Univ. Valle 2(1/2):1-17.

CANAL DAZA, N., ZUCCHI, R.A., SILVA, N.M. \& SILVEIRA NETO, S. 1995. Análise faunística dos parasitóides (Hymenoptera: Braconidae) de Anastrepha (Dip., Tephritidae) em Manaus e Iranduba, Estado do Amazonas. Acta Amaz. 25(3/4):235-246.

CARVALHO, R.S. \& MALAVASI, A. 2003. Monitoramento de parasitóides nativos de moscas-das-frutas (Tephritidae) antes da liberação de Diachasmimorpha longicaudata na região Amazônica. Embrapa Mandioca e Fruticultura, Cruz das Almas, 8p. Embrapa Mandioca e Fruticultura, Comunicado técnico, 96.

CARVALHO, R.S. 2002. Impacto da introdução do braconídeo exótico Diachasmimorpha longicaudata no Recôncavo Baiano, no Semi-árido e na Amazônia e seu potencial de uso no Brasil. In Anais do $19^{\circ}$ Congresso Brasileiro de Entomologia. Sociedade Entomólogica do Brasil, Manaus. CD-ROM.

CARVALHO, R.S. 2003. Estudos de laboratório e de campo com o parasitóide exótico Diachasmimorpha longicaudata Ashmead (Hymenoptera: Braconidae) no Brasil. Tese de Doutorado, Instituto de Biociências, Universidade de São Paulo, São Paulo, 182p.

COSTA, S.G.M., QUERINO, R.B., RONCHI-TELES, B., PENTEADO-DIAS, A.M.M. \& ZUCCHI, R.A. 2009. Parasitoid diversity (Hymenoptera: Braconidae and Figitidae) on frugivorous larvae (Diptera: Tephritidae and Lonchaeidae) at Adolpho Ducke Forest Reserve, Central Amazon Region, Manaus, Brazil. Brazil. J. Biol. 69(2):363-370.

LEONEL Jr., F.L., ZUCCHI, R.A., WHARTON, R.A. 1995. Distribution and tephritidae host (Diptera) of braconidae parasitoids (Hymenoptera) in Brazil. Int. J. Pest Manag. 41(4)208-213.
NASCIMENTO, A.S., CARVALHO, R.S. \& MALAVASI, A. 2000. Monitoramento populacional. In Moscas-das-frutas de importância econômica no Brasil: conhecimento básico e aplicado (A. Malavasi \& R.A. Zucchi, eds). São Paulo, Holos, p. 109-112.

RONCHI-TELES, B. 2000. Ocorrência e flutuação populacional de espécies de moscas-das-frutas e parasitóides com ênfase para o gênero Anastrepha (Diptera: Tephritidae) na Amazônia Brasileira. Tese de Doutorado, Instituto Nacional de Pesquisas da Amazônia, Universidade do Amazonas, Manaus, 156p.

SILVA, N.M. \& RONCHI-TELES, B. 2000. Amapá, Amazonas, Pará, Rondônia e Roraima. In Moscas-das-frutas de importância econômica no Brasil: conhecimento básico e aplicado (A. Malavasi \& R.A. Zucchi, eds). São Paulo, Holos, p. 203-209.

SILVA, N.M. 1993.Levantamento e análise faunística de moscas-das-frutas (Diptera: Tephritidae) em quatro locais do Estado do Amazonas. Tese de Doutorado, Escola Superior de Agricultura Luiz de Queiroz, Universidade de São Paulo, Piracicaba, 152p.

SILVA, R.A., XAVIER, S.L.O., SOUZA FILHO, M.F., SILVA, W.R., NASCIMENTO, D.B. \& DEUS, E.G. 2007. Frutíferas hospedeiras e parasitóides (Hym., Braconidae) de Anastrepha spp. (Dip., Tephritidae) na Ilha de Santana, Estado do Amapá, Brasil. Arq. Instit. Biol. 74:153-156.

THOMAZINI, M.J. \& ALBUQUERQUE, E.S. 2009. Parasitóides (Hymenoptera: Braconidae) de Anastrepha Schiner (Diptera: Tephritidae) no estado do Acre. Acta Amaz. 39(1):245-248.

THOMAZINI, M.J., ALBUQUERQUE, E.S. \& SOUZA-FILHO, M.F. 2003. Primeiro registro de espécies de Anastrepha (Diptera: Tephritidae) no estado do Acre. Neotrop. Entomol. 32(4):723-724.

ZUCCHI, R.A. 2008. Fruit flies in Brazil - Anastrepha species and their hosts plants. http://www.lea.esalq.usp.br/anastrepha/ (último acesso em 20/01/2010).

Recebido em 26/01/2010

Versão reformulada recebida em 07/04/2010

Publicado em 01/07/2010 